\title{
Identification and quantification of phenolic compounds in bambangan (Mangifera pajang Kort.) peels and their free radical scavenging activity.
}

\begin{abstract}
Phenolic compounds and antioxidant capacity of acidified methanolic extract prepared from fully ripe bambangan (Mangifera pajang K.) peel cultivated in Sarawak, Malaysia, were analyzed. The total phenolic content ( $98.3 \mathrm{mg} \mathrm{GAE} / \mathrm{g}$ ) of bambangan peel powder (BPP) was determined by the Folin-Ciocalteu method. BPP showed a strong potency of antioxidant activity and was consistent with that of BHT and vitamin $\mathrm{C}$ as confirmed by the DPPH (1,1diphenyl-2-picrylhydrazyl) radical scavenging activity and FRAP (ferric-reducing antioxidant power) assays. Gallic acid, p-coumaric acid, ellagic acid, protocatechuic acid, and mangiferin were the major compounds among the 16 phenolics that have been identified and quantified in M. pajang peels with 20.9, 12.7, 7.3, 5.4, and $4.8 \mathrm{mg} / \mathrm{g}$ BPP, respectively. Peak identities were confirmed by comparing their retention times, UV-vis absorption spectra, and mass spectra with authentic standards. The 16 phenolic compounds identified in M. pajang K. using HPLC-DAD and TSQ-ESI-MS are reported here for the first time.
\end{abstract}

Keyword: HPLC-ESI-MS; Mangifera pajang; Peel; Phenolic compounds; Scavenging activity. 